\title{
Ischemic patterns assessed by positron emission tomography predict adverse outcome in patients with idiopathic dilated cardiomyopathy
}

Richard M. de Jong, MD, PhD, ${ }^{a}$ Rene A. Tio, $M D, P h D,{ }^{a}$ Pim van der Harst, $M D$, PhD, ${ }^{a}$ Adriaan A. Voors, MD, PhD, ${ }^{a}$ Paul M. Koning, BSc, ${ }^{a}$

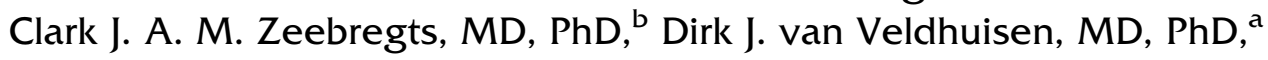
Rudi A. J. O. Dierckx, MD, PhD, ${ }^{c}$ and Riemer H. J. A. Slart, MD, PhD ${ }^{c}$

Background. Although patients with idiopathic dilated cardiomyopathy (DCM) have no coronary artery disease, regional impairment of myocardial perfusion combined with preserved metabolism has been found using positron emission tomography (PET). Our aim was to assess the prognostic relevance of PET-mismatch between stress myocardial perfusion and glucose uptake on clinical outcome in DCM.

Methods. In 24 patients with DCM who underwent both myocardial perfusion and metabolism PET scanning, "mismatch" was assessed and the association with clinical outcome (hospitalization, mortality, and heart transplantation) was investigated.

Results. Mismatch was found in 16 patients $(66.7 \%)$. Univariate analysis showed that the presence of mismatch was associated with adverse outcome $(P=0.03)$. After adjustment for sex and age, the association remained significant with an adjusted relative risk of $10.4(95 \% \mathrm{CI}$ 1.1-103; $P=0.04$ ) for death, heart transplant, or hospitalization. Univariate analysis also showed that a higher extent of mismatch was significantly associated with adverse outcome $(P=0.02)$. After adjusting for sex and age, the association remained significant with an adjusted relative risk of $6.5[95 \%$ CI $1.2-36 ; P=0.03]$ for death, heart transplantation, or hospitalization.

Conclusion. PET stress perfusion-metabolism mismatch, indicative for ischemia, is frequently found in DCM patients and related to a poorer outcome. (J Nucl Cardiol 2009;16:769-74.)

Key Words: Heart failure $\cdot$ dilated cardiomyopathy $•$ positron emission tomography • myocardial perfusion $\cdot$ myocardial ischemia

In idiopathic dilated cardiomyopathy (DCM), it has generally been assumed that myocardial ischemia does not play a role since the coronary arteries show by definition no signs of significant stenoses. However,

From the Thoraxcenter, Department of Cardiology, ${ }^{\mathrm{a}}$ Department of Surgery, Division of Vascular Surgery, ${ }^{\mathrm{b}}$ and Department of Nuclear Medicine and Molecular Imaging, ${ }^{\text {c }}$ University Medical Centre Groningen, Groningen, The Netherlands.

Received for publication Feb 2, 2009; final revision accepted Jul 16, 2009.

Reprint requests: Richard M. de Jong, MD, PhD, Thoraxcenter, Department of Cardiology, University Medical Centre Groningen, Hanzeplein 1, P.O. Box 30.001, 9700 RB Groningen, The Netherlands; R.M.de.Jong@thorax.umcg.nl.

$1071-3581 / \$ 34.00$

Copyright (c) 2009 The Author(s). This article is published with open access at Springerlink.com

doi:10.1007/s12350-009-9130-9 some studies have suggested that impaired endothelial function and perfusion abnormalities do occur in DCM. ${ }^{1,2}$ Regional ischemia can be investigated with positron emission tomography (PET), looking at a mismatch between stress myocardial perfusion and FDG metabolism. ${ }^{3}$ FDG is a memory marker of transient ischemia and thus regional myocardial uptake of FDG can be measured at least 1 hour and in some patients up to 24 hours after stress to assess ischemia. ${ }^{4,5}$ Using PET, our center has demonstrated that areas of ischemia may be present in a substantial percentage of patients with DCM. ${ }^{6}$ These areas were associated with high left ventricular wall stress and a regional switch to an anaerobic metabolism. Furthermore, long-term treatment with betablockers can increase myocardial perfusion reserve ${ }^{7}$ and reduce stress-induced perfusion defects. ${ }^{8}$ Thus far, the relevance and pathophysiological 
role of ischemia in DCM is unknown. The ischemic regions may be the result of cardiac dysfunction. This might be explained by a reduced cardiac output leading to myocardial ischemia, especially during stress, which may lead to cardiac dysfunction in a vicious circle. Alternatively, these regions may be related to changes associated with DCM such as endothelial dysfunction, decreased angiogenesis, and impaired perfusion. ${ }^{9}$ Since PET studies suggest regions of chronic ischemia in DCM, it is tempting to draw parallels with ischemic heart disease. Ischemia and hibernation in heart failure due to coronary heart disease predict a higher mortality rate if left untreated and shows substantial benefit from revascularisation therapy in terms of prognosis..$^{10,11}$ The aim of this study was to assess the potential relevance of regional ischemia identified by PET on clinical outcome in DCM.

\section{METHODS}

\section{Patient Population and Study Design}

Twenty-four patients with DCM were identified with both myocardial perfusion and glucose uptake PET scans available. The data from 22 of these patients were described in an earlier publication of our center. ${ }^{6}$ Underlying causes for heart failure (e.g., hypertension, valvular disease, and diabetes) were excluded using clinical history, physical examination, blood chemistry, echocardiography, and coronary angiography. Additional endomyocardial biopsy was performed in 15 patients and did not reveal a cause for left ventricular dysfunction. Echocardiography was used to assess left ventricular end diastolic dimension (LVEDD) and left ventricular ejection fraction (LVEF). Peak oxygen consumption $\left(\mathrm{VO}_{2} \mathrm{max}\right)$ was obtained in 22 patients (2 patients were not able to exercise adequately).

\section{PET Study Protocol}

All PET scans were performed after withdrawal of vasoactive medications including diuretics, ACE-inhibitors, beta-blockers, calcium-channel blockers, statins, and long acting nitrates at least 24 hours before the PET study. PET scans were performed on a 951 Siemens (ECAT) positron camera (Siemens AG, Knoxfille, TN), which images 31 planes simultaneously over $10.8 \mathrm{~cm}$ (axial field of view). Positioning of the subjects was done with the aid of a rectilinear scan. Photon attenuation was measured using an external ring source filled with ${ }^{68} \mathrm{Ge} /{ }^{68} \mathrm{Ga}$ and data were automatically corrected for accidental coincidence and dead time. Patients were continuously monitored with 12-lead electrocardiography and blood pressure was obtained every 10 minutes. During pharmacologically induced stress, blood pressure was measured every minute. Myocardial perfusion was measured according to the methods described by Schelbert et al ${ }^{12}$ using ${ }^{13} \mathrm{NH}_{3}$ as a perfusion tracer and dipyridamol as the pharmacological stress agent. After injection of ${ }^{13} \mathrm{NH}_{3}(370 \mathrm{MBq})$ dynamic rest imaging was performed for 15 minutes (frames $12 \times 10$ seconds, $1 \times 2$ minutes, $1 \times 4$ minutes, $1 \times 7$ minutes). Dipyridamol stress imaging was performed identically after dipyridamol infusion 6 minutes prior to the injection of ${ }^{13} \mathrm{NH}_{3}$. Myocardial glucose uptake was studied according to the methods described by Choi et al ${ }^{13}$ using ${ }^{18} \mathrm{FDG}$ as tracer. ${ }^{18} \mathrm{FDG}$ was injected 20 minutes after the last ${ }^{13} \mathrm{NH}_{3}$ injection. To ensure sufficient myocardial glucose uptake, patients were either given $75 \mathrm{~g}$ of glucose orally or the glucose clamping technique was applied. ${ }^{18} \mathrm{FDG}$ imaging was performed 5 minutes after injection of ${ }^{18} \mathrm{FDG}(200 \mathrm{MBq})$ and continued for 35 minutes (frames $8 \times 15$ seconds, $4 \times 30$ seconds, $1 \times 1$ minutes, $1 \times 5$ minutes, $1 \times 10$ minutes, $1 \times 15$ minutes).

\section{Analysis of PET Data}

The analysis of PET data has been extensively described in a previous publication. ${ }^{3}$ In brief, all data were reoriented to 10 short-axis images. Each of the 10 slices was divided into 48 segments $\left(7.5^{\circ}\right.$ each). For each individual, segment myocardial perfusion and glucose uptake were calculated. From these data, a parametric polar map for each modality was constructed. To calculate mismatch, we used the dipyridamol-derived perfusion. Quantification of mismatch was performed by first normalizing the ${ }^{18}$ FDG uptake polar map and the dipyridamol perfusion polar map to their means. Then, a difference polar map was created by subtracting the normalized dipyridamol perfusion polar map from the normalized ${ }^{18} \mathrm{FDG}$ uptake polar map. Mismatch for each of the 480 segments was calculated as the percentage myocardium above the $95 \%$ confidence interval (CI) of a normal data base and results were expressed as percentage of the total myocardium.

\section{Follow-up}

Patients were contacted by telephone and interviewed and medical records were reviewed. The primary endpoint was defined as cardiac death, heart transplantation, or hospitalization for progressive heart failure. Cardiac death was ascertained by medical records. Follow-up was completed in all 24 patients.

\section{Statistical Analysis}

Data are expressed as the mean \pm standard deviation and Student's $t$-test was used to compare the means of continuous variables. Univariate analyses of cumulative event rates for different groups were estimated using the Kaplan-Meier method. A multivariate analysis including sex, age and PET mismatch between stress myocardial perfusion and glucose uptake was performed using Cox regression techniques. Statistical analysis was performed using SPSS, version 12.0.

\section{RESULTS}

\section{Patient Characteristics}

The study population consisted of 24 patients and their characteristics are shown in Table 1. Age was 
Table 1. Clinical characteristics of all patients and patients with and without mismatch measured by PET

\section{Total Group 1, no mismatch Group 2, mismatch P, Group $(n=24)$ $(n=8) \quad(n=16)$ vs 2}

\begin{tabular}{|c|c|c|c|c|}
\hline \multicolumn{5}{|l|}{ Clinical data } \\
\hline Age (years) & 49 & 54 & 46 & 0.19 \\
\hline Men (\%) & 59 & 67 & 50 & 0.44 \\
\hline $\begin{array}{l}\text { Systolic blood pressure } \\
(\mathrm{mmHg})\end{array}$ & 109 & 110 & 108 & 0.76 \\
\hline $\begin{array}{l}\text { Diastolic blood pressure } \\
(\mathrm{mmHg})\end{array}$ & 64 & 66 & 63 & 0.35 \\
\hline Heart rate (/minutes) & 74 & 72 & 75 & 0.65 \\
\hline \multicolumn{5}{|l|}{ LV function data } \\
\hline LVEDD* $(m m)$ & 66 & 64 & 66 & 0.58 \\
\hline LVEF* * (\%) & 32 & 32 & 34 & 0.81 \\
\hline $\mathrm{VO}_{2} \max (\mathrm{mL} / \mathrm{kg} /$ minutes $)$ & 21 & 21 & 21 & 0.98 \\
\hline
\end{tabular}

Mean values: * left ventricular end diastolic diameter, ${ }^{*}$ left ventricular ejection fraction.

$49 \pm 14$ years and $14(58 \%)$ patients were men. Mean ejection fraction was $32 \pm 14 \%$, and mean LVEDD was $66 \pm 7 \mathrm{~mm}$. Average $\mathrm{VO}_{2} \max$ was $21.6 \pm 4.4$ $\mathrm{mL} / \mathrm{kg} /$ minutes.

\section{PET Data}

Resting myocardial perfusion was $106 \pm 24$ $\mathrm{mL} /$ minutes per $100 \mathrm{~g}$ and dipyridamol myocardial perfusion was $186 \pm 45 \mathrm{~mL} /$ minutes per $100 \mathrm{~g}$. Total ${ }^{18} \mathrm{FDG}$ uptake was $56 \pm 18 \mu \mathrm{mol} /$ minutes per 100 grams. Stress perfusion-metabolism mismatch was found in 16 patients $(67 \%)$, with a mean of $14 \%$ (range $4.5-32 \%)$ of the left ventricular myocardium.

\section{Mismatch and Survival}

Mean duration of follow-up was 5.5 years during which 10 patients $(42 \%)$ reached the primary endpoint; seven patients died, two patients received heart transplantation, and one patient was hospitalized for progressive heart failure.

Clinical outcome in patients with $(n=16)$ vs patients without mismatch $(n=8)$ was analysed and nine events $(56 \%)$ in patients with mismatch and one event $(13 \%)$ in patients without mismatch were found. Univariate Kaplan-Meier survival analysis with log-rank statistics showed a significant better outcome in patients without mismatch $(P=0.03)$ (Figure 1). Adjusting for sex and age with Cox-proportional hazard showed a hazard ratio of 10.4 (95\% CI 1.1-103, $P=0.04$ ). The hazard ratio for age was $1.0(95 \% \mathrm{CI} 0.97-1.1, P=0.34)$ and sex 2.0 (95\% CI 0.56-7.1, $P=0.29$ ).

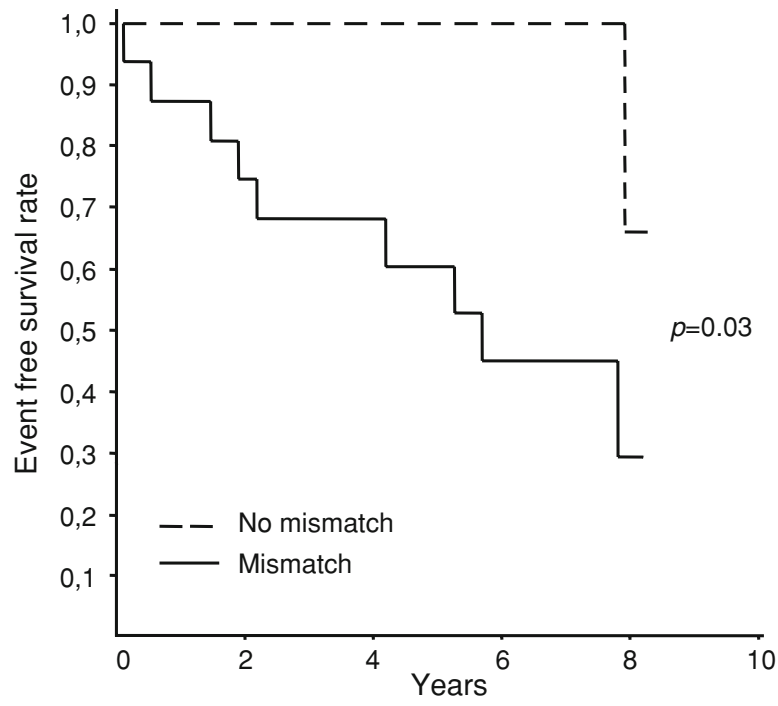

Figure 1. Kaplan-Meier survival according to the presence of mismatch.

In order to determine whether the extent of PET stress perfusion-metabolism mismatch influences clinical outcome, patients were ranked into two groups according to the median percentage of mismatch. In patients below the median percentage of mismatch, two events occurred, compared to seven events in patients above the median value. Univariate survival analysis showed a significant better outcome in patients below the median $(P=0.02)$ (Figure 2). Multivariate analysis with adjustment for sex and age showed a hazard ratio of 6.5 (95\% CI 1.2-36, $P=0.03$ ). The hazard ratio for age was $1.0(95 \%$ CI $0.98-1.1, P=0.34)$ and sex $1.5(95 \%$ CI $0.42-5.6, P=0.29)$. 


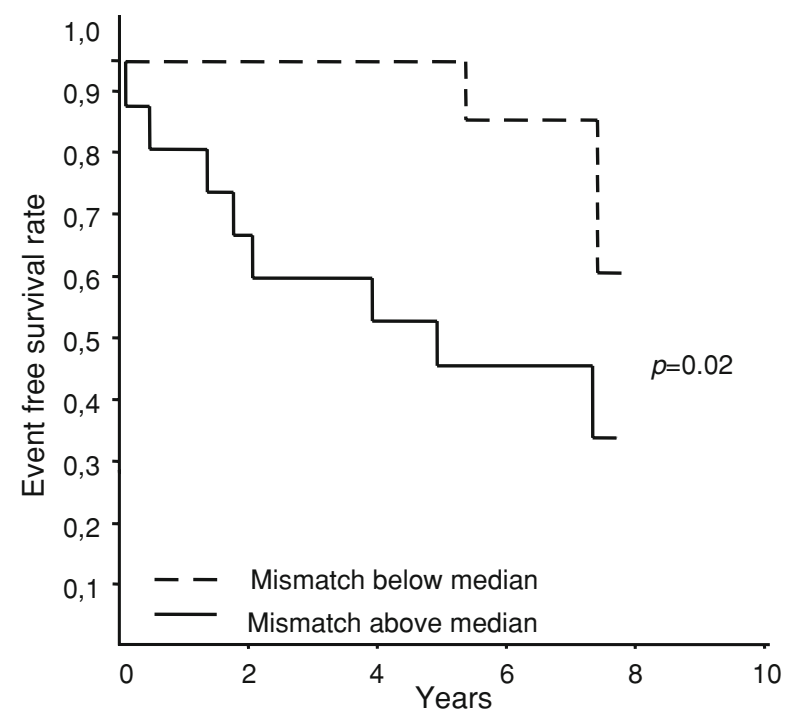

Figure 2. Kaplan-Meier survival in patients according to the percentage of mismatch.

\section{DISCUSSION}

The present study is the first to demonstrate an association between myocardial PET stress perfusionmetabolism mismatch and clinical outcome in patients with DCM.

There is convincing evidence that in ischemic heart disease, a deficient oxygen supply as a result of coronary artery disease is the primary cause for myocardial dysfunction, leading to cardiac remodeling and ultimately heart failure ${ }^{14}$ However, the opposite may also be true as there is increasing evidence that left ventricular dysfunction and heart failure cause coronary perfusion abnormalities and myocardial ischemia, which may in turn result in further myocardial dysfunction. Unverferth et $\mathrm{al}^{15}$ were the first to propose possible involvement of ischemia in the pathophysiology of DCM and since then several studies have shown myocardial perfusion and metabolism abnormalities in DCM. We previously demonstrated that regional stress perfusion-metabolism mismatch occurs in patients with DCM. ${ }^{6}$ These regions showed an additional switch from aerobic to an anaerobic metabolism; findings comparable with chronic myocardial ischemia in ischemic cardiomyopathy. These perfusion abnormalities may have multiple explanations. First, dilation of the ventricles results in a decrease of capillary density, thereby increasing the distances over which oxygen has to diffuse. A study from Abraham et al showed a $66 \%$ decrease in capillary density in patients with DCM. ${ }^{16}$ This decrease in capillary density may in part be explained by a defective angiogenesis. This fundamental process by which new blood vessels are formed is characterized by a precise interplay among multiple pro- and anti-angiogenic molecules, and at least one of these angiogenic factors, vascular endothelial growth factor (VEGF), was shown to be selectively down regulated in DCM. ${ }^{17}$ However, much remains unknown about capillary and VEGF expression in DCM. Other pathophysiological changes occurring in the failing heart that impair myocardial perfusion include myocardial fibrosis and matrix apposition. ${ }^{18}$ Also, arteriolar and capillary diameter and swelling in DCM have been reported. ${ }^{19}$ Finally, models of DCM in Syrian hamsters showed microcirculatory defects caused by microvascular spasms and endothelial dysfunction leading to myocytolysis and ischemia. $^{20}$

The PET stress perfusion-metabolism mismatch characteristics seen in our population strongly resemble ischemia of ischemic heart disease. Chronic ischemia might also lead to hibernation, but this has not been investigated in our patients since mismatch was assessed between myocardial perfusion during stress and glucose uptake directly after stress. Alternatively, alterations of myocardial substrate metabolism may occur as a result of heart failure without signs of ischemia. Fatty acid metabolism in patients with DCM is regionally impaired and a relation with prognosis has recently been found using nuclear imaging with ${ }^{123}$ I-betamethyl-p-iodophenyl-pentadecanoic acid combined with ${ }^{201} \mathrm{Tl}^{21}$ Moreover, a study using ${ }^{201} \mathrm{Tl}$ with single photon emission computed tomography and ${ }^{18}$ FDG PET in patients with DCM reported mismatch areas that were related to prognosis, which is consistent with the results of our study. ${ }^{22}$ Furthermore, functional impairment assessed by dobutamine stress echocardiography in DCM suggests hibernation; $69 \%$ of the patients with DCM show a decrease in wall motion during induced stress. ${ }^{23}$ These findings are similar to ischemic contractile responses in patients with coronary artery disease during dobutamine stress echocardiography. Because of these similarities, it is tempting to draw parallels between ischemic myocardium in DCM and ischemic cardiomyopathy. The pathological concept of myocardial hibernation was first proposed by Rahimtoola ${ }^{24}$ to characterize a situation of "prolonged subacute or chronic ischemia in which myocardial contractility and metabolism and ventricular function are reduced to match the reduced blood supply which is a new state of equilibrium whereby myocardial necrosis is prevented, and the myocardium is capable of restoring and returning to normal or near-normal function on restoration of an adequate blood supply." In ischemic heart disease, areas of noncontractile, but viable myocardium are important as patients show tremendous benefit from cardiac revascularisation therapy over patients without hibernating myocardium. ${ }^{25}$ Obviously, ischemic cardiomyopathy is caused by coronary artery disease, and restoring myocardial perfusion in 
DCM will not benefit from revascularisation therapy in terms of coronary bypass grafting since coronaries are normal by definition and perfusion abnormalities occur at the level of arterioles and capillaries.

Higher mortality in our DCM patients with PET mismatch or chronic ischemia may, in theory, have two explanations. First, primary myocardial dysfunction results in myocardial remodeling, which predispose to perfusion abnormalities. ${ }^{26}$ The resulting ischemia further impairs cardiac function, and so on. This suggests a reciprocal relation between myocardial dysfunction and ischemia, resulting in accelerated disease progression. ${ }^{27}$ Second, multiple regional adaptive responses due to chronic ischemia may lead to electrically unstable substrate with increased risk of sudden cardiac death. ${ }^{28}$ Thus, the higher mortality may partially be explained by hibernating myocardium which is likely to accelerate disease progression and is prone to develop potentially lethal arrhythmias. Both may be present simultaneously and might explain the observed high rate of mortality in our patients with mismatch.

Given the relation between mismatch and prognosis found in our study and the observed abnormalities in microvessels in DCM, one could speculate that therapeutic interventions using, e.g., VEGF or erythropoietin to stimulate vascular growth in ischemic regions ${ }^{29}$ might have a positive effect on prognosis for patient with heart failure due to DCM. ${ }^{30}$

This study has some limitations which merit consideration. The study was retrospective and therefore the results require confirmation in prospective investigations. A limited number of patients was studied and although a significant relation could be found between mismatch and clinical outcome, this has to be confirmed in larger patient groups. Noteworthy, in our study standard parameters like peak $\mathrm{VO}_{2}$ did not predict mortality while perfusion-metabolism did. This might be explained by the fact that our study was relatively small or that the range of standard parameters in our patient groups is limited. The fact that stress perfusion-metabolism mismatch did predict mortality might suggest that this parameter is more powerful than the standard parameters that predict mortality. Parameters, like NT-proBNP, that are now known to be of predictive value were not measured in our patients.

\section{CONCLUSION}

This study showed that in patients with DCM, PET stress perfusion-metabolism mismatch is frequently found and significantly associated with clinical outcome. Larger prospective studies are needed to confirm these results and further studies are required to investigate the role of mismatch as a pathophysiological substrate for cardiac dysfunction and disease progression in patients with DCM.

\section{Open Access}

This article is distributed under the terms of the Creative Commons Attribution Noncommercial License which permits any noncommercial use, distribution, and reproduction in any medium, provided the original author(s) and source are credited.

\section{References}

1. Treasure CB, Vita JA, Cox DA, et al. Endothelium-dependent dilation of the coronary microvasculature is impaired in dilated cardiomyopathy. Circulation 1990;81:772-9.

2. Juilliere Y, Marie PY, Danchin N, et al. Radionuclide assessment of regional differences in left ventricular wall motion and myocardial perfusion in idiopathic dilated cardiomyopathy. Eur Heart J 1993;14:1163-9.

3. Blanksma PK, Willemsen AT, Meeder JG, et al. Quantitative myocardial mapping of perfusion and metabolism using parametric polar map displays in cardiac PET. J Nucl Med 1995;36: 153-8.

4. Abbott BG, Liu YH, Arrighi JA. [18F]Fluorodeoxyglucose as a memory marker of transient myocardial ischaemia. Nucl Med Commun 2007;28:89-94.

5. Dou KF, Yang MF, Yang YJ, Jain D, He ZX. Myocardial 18F-FDG uptake after exercise-induced myocardial ischemia in patients with coronary artery disease. J Nucl Med 2008;49: 1986-91.

6. van den Heuvel AF, van Veldhuisen DJ, van der Wall EE, et al. Regional myocardial blood flow reserve impairment and metabolic changes suggesting myocardial ischemia in patients with idiopathic dilated cardiomyopathy. J Am Coll Cardiol 2000;35:19-28.

7. Erdogan D, Gullu H, Caliskan M, et al. Nebivolol improves coronary flow reserve in patients with idiopathic dilated cardiomyopathy. Heart 2007;93:319-24.

8. Neglia D, De Maria R, Masi S, et al. Effects of long-term treatment with carvedilol on myocardial blood flow in idiopathic dilated cardiomyopathy. Heart 2007;93:808-13.

9. De Boer RA, Pinto YM, van Veldhuisen DJ. The imbalance between oxygen demand and supply as a potential mechanism in the pathophysiology of heart failure: The role of microvascular growth and abnormalities. Microcirculation 2003;10:113-26.

10. Di Carli MF, Asgarzadie F, Schelbert HR, et al. Quantitative relation between myocardial viability and improvement in heart failure symptoms after revascularization in patients with ischemic cardiomyopathy. Circulation 1995;92:3436-44.

11. Senior R, Kaul S, Raval U, Lahiri A. Impact of revascularization and myocardial viability determined by nitrate-enhanced Tc- $99 \mathrm{~m}$ sestamibi and Tl-201 imaging on mortality and functional outcome in ischemic cardiomyopathy. J Nucl Cardiol 2002;9:454-62.

12. Schelbert HR, Phelps ME, Huang SC, et al. N-13 ammonia as an indicator of myocardial blood flow. Circulation 1981;63:1259-72.

13. Choi Y, Hawkins RA, Huang SC, et al. Parametric images of myocardial metabolic rate of glucose generated from dynamic cardiac PET and 2-[18F]fluoro-2-deoxy-D-glucose studies. J Nucl Med 1991;32:733-8.

14. Sharpe N. Cardiac remodeling in coronary artery disease. Am J Cardiol 2004;93:17B-20B.

15. Unverferth DV, Magorien RD, Lewis RP, Leier CV. The role of subendocardial ischemia in perpetuating myocardial failure in 
patients with nonischemic congestive cardiomyopathy. Am Heart J 1983;105:176-9.

16. Abraham D, Hofbauer R, Schafer R, et al. Selective downregulation of VEGF-A(165), VEGF-R(1), and decreased capillary density in patients with dilative but not ischemic cardiomyopathy. Circ Res 2000;87:644-7.

17. De Boer RA, Henning RH, Tio RA, et al. Identification of a specific pattern of downregulation in expression of isoforms of vascular endothelial growth factor in dilated cardiomyopathy. Heart 2002;88:412-4.

18. Colucci WS. Molecular and cellular mechanisms of myocardial failure. Am J Cardiol 1997;80:15L-25L.

19. Mosseri M, Schaper J, Admon D, et al. Coronary capillaries in patients with congestive cardiomyopathy or angina pectoris with patent main coronary arteries. Ultrastructural morphometry of endomyocardial biopsy samples. Circulation 1991;84:203-10.

20. Figulla HR, Vetterlein F, Glaubitz M, Kreuzer H. Inhomogenous capillary flow and its prevention by verapamil and hydralazine in the cardiomyopathic Syrian hamster. Circulation 1987;76:208-16.

21. Inoue A, Fujimoto S, Yamashina S, Yamazaki J. Prediction of cardiac events in patients with dilated cardiomyopathy using 123IBMIPP and 201Tl myocardial scintigraphy. Ann Nucl Med 2007; 21:399-404.

22. Isobe $\mathrm{S}$, Ando A, Nanasato M, et al. Combined study with FDG PET and Tl SPECT in patients with idiopathic dilated cardiomyopathy. Nucl Med Commun 2003;24:1071-80.

23. de Jong RM, Cornel JH, Crijns HJ, van Veldhuisen DJ. Abnormal contractile responses during dobutamine stress echocardiography in patients with idiopathic dilated cardiomyopathy. Eur J Heart Fail 2001;3:429-36.

24. Rahimtoola SH. A perspective on the three large multicenter randomized clinical trials of coronary bypass surgery for chronic stable angina. Circulation 1985;72:V123-35.

25. Bax JJ, Cornel JH, Visser FC, et al. Prediction of improvement of contractile function in patients with ischemic ventricular dysfunction after revascularization by fluorine- 18 fluorodeoxyglucose single-photon emission computed tomography. J Am Coll Cardiol 1997;30:377-83.

26. Udelson JE, Konstam MA. Relation between left ventricular remodeling and clinical outcomes in heart failure patients with left ventricular systolic dysfunction. J Card Fail 2002;8:S465-71.

27. van Veldhuisen DJ, van den Heuvel AF, Blanksma PK, Crijns HJ. Ischemia and left ventricular dysfunction: A reciprocal relation? J Cardiovasc Pharmacol 1998;32:S46-51.

28. Canty JM Jr, Suzuki G, Banas MD, Verheyen F, Borgers M, Fallavollita JA. Hibernating myocardium: Chronically adapted to ischemia but vulnerable to sudden death. Circ Res 2004;94:1142-9.

29. Westenbrink BD, Oeseburg H, Kleijn L, et al. Erythropoietin stimulates normal endothelial progenitor cell-mediated endothelial turnover, but attributes to neovascularization only in the presence of local ischemia. Cardiovasc Drugs Ther 2008;22:265-74.

30. Belonje AM, Voors AA, van Gilst WH, van Veldhuisen DJ. Erythropoietin in chronic heart failure. Congest Heart Fail 2007; 13:289-92. 